\title{
Multiplierless Wideband and Narrowband CIC Compensator for SDR Application
}

\author{
Gordana Jovanovic Dolecek \\ Department of Electronics, Institute INAOE, Puebla, Mexico \\ Email: gordana@ieee.org
}

How to cite this paper: Dolecek, G.J. (2017) Multiplierless Wideband and Narrowband CIC Compensator for SDR Application. Int. J. Communications, Network and System Sciences, 10, 19-26. https://doi.org/10.4236/ijcns.2017.108B003

Received: March 20, 2017

Accepted: August 11, 2017

Published: August 14, 2017

\begin{abstract}
This paper presents multiplierless CIC compensator for software-defined radio (SDR) application. The compensator is composed of two simple filters with sinewave form of magnitude responses. The parameters of the design are the sinewave amplitudes expressed as powers-of-two and estimated in a way to fulfill the absolute value of the maximum passband deviation of $0.25 \mathrm{~dB}$ and $0.05 \mathrm{~dB}$, for the wideband and narrowband compensations, respectively. The proposed compensator requires maximum nine adders. The comparisons with the methods proposed in literature show the benefits of the proposed compensator.
\end{abstract}

\section{Keywords}

Software Radio, Sampling Rate Conversion, Decimation, CIC Filter, Compensator

\section{Introduction}

Software-defined radio (SDR) has found important role in modern wireless communications. The main idea in SDR is to move the analog-to-digital converters (ADCs) and digital-to-analog converters (DACs) as close as possible to the antenna and thus perform all signal processing in the digital form [1]. As a consequence, SDR is able to support different wireless standards using the same hardware. Knowing that different wireless standards require different sampling rate for baseband processing, the sampling-rate conversion (SRC) becomes a key operation in a SDR receiver [2].

SRC involves resampling in a digital domain thus causing aliasing and imaging which must be eliminated by filtering [3]. CIC (cascaded-integrator-comb) filter proposed in [4] is widely used as anti-aliasing and anti-imaging filter due to its simplicity: the filter requires no multiplication or coefficient storage. The 
transfer function of the CIC decimation filter in $z$-domain is given as:

$$
H(z)=\left[\frac{1}{M} \frac{1-z^{-M}}{1-z^{-1}}\right]^{K},
$$

where $M$ is the decimation factor and $K$ is the number of the cascaded filters.

However, its magnitude characteristic:

$$
\left|H\left(e^{j \omega}\right)\right|=\left|\frac{\sin (\omega M / 2}{M \sin (\omega / 2)}\right|^{K},
$$

exhibits a low attenuation in the stopband of interest and a passband droop in the band of interest. As $K$ increases, the stopband attenuation increases, resulting in an increased droop in the passband, which may deteriorate the decimated signal. The motivation of this work is to achieve good CIC wideband and narrowband compensation while keeping low rate of addition operations.

Different methods were proposed to compensate for the CIC passband droop. The compensators which need multipliers were proposed for example in [5] [6] [7]. However, due to lower power consumption, multiplierless compensators [8]-[13] are of more interest for SDR application. A two/stage CIC compensator with sinewave form of magnitude responses was recently proposed in [13]. The parameters of design are amplitudes of sinewaves expressed as sum-of-powersof-two (SPT), and chosen in a way to provide better compensation than any other multiplierless compensator from literature. (The absolute value of the maximum passband deviation of the compensated comb is less than $0.1 \mathrm{~dB}$ ). The compensator requires 11 adders for $K=2,4$, and 5, and 10 adders for $K=3$, and 6 . The goal here is to design a compensator requiring even fewer adders than the compensator in [13] while permitting a slight increase of the absolute value of the maximum passband deviation. The method is based on the sinewave magnitude responses multiplierless filters.

The paper is organized in the following way. Next section introduces transfer function of the proposed filter and describes the choice of the design parameters for wideband and narrowband compensation. Some comparisons are provided in Section 3.

\section{Proposed Compensator}

\subsection{Transfer Function of Proposed Compensator}

Like compensator in [13], the proposed compensator has magnitude response in the form:

$$
\begin{gathered}
\left|G_{c}\left(e^{j \omega M}\right)\right|=\left|G_{1}\left(e^{j \omega M}\right)\right| \times\left|G_{2}\left(e^{j \omega M}\right)\right|, \\
\left|G_{1}\left(e^{j \omega M}\right)\right|=1+B_{1} \sin ^{4}(\omega M / 2) . \\
\left|G_{2}\left(e^{j \omega M}\right)\right|=1+B_{2} \sin ^{2}(\omega M / 2) .
\end{gathered}
$$

In contrast to the method in [13], we express the parameters of sinusoidal functions as powers of two, in order to decrease the number of the required adders: 


$$
B_{1}=2^{-N_{1}} ; B_{2}=2^{-N_{2}} .
$$

where $N_{1}$ and $N_{2}$ are integers.

The corresponding transfer function at low rate becomes:

$$
G_{c}(z)=G_{1}(z) G_{2}(z),
$$

where

$$
G_{1}(z)=2^{-4} 2^{-N_{1}} \times\left[1+z^{-4}-4\left(z^{-1}+z^{-3}\right)+\left(2^{2}+2\right) z^{-2}\right]+z^{-2},
$$

and

$$
G_{2}(z)=2^{-2}\left[\left(-1+2 z^{-1}-z^{-2}\right) 2^{-N_{2}}+2^{2} z^{-1}\right] .
$$

As a result, filters (8) and (9) require 6 and 3 adders, respectively, i.e. the compensator (7) requires total of 9 adders.

\subsection{Wideband Compensation}

We consider the passband edge $\omega_{p}=\pi /(2 M)$, and impose the following condition:

$$
\max _{N_{1}, N_{2}}\left\{20 \log 10\left|H\left(e^{j \omega}\right) G_{1}\left(e^{j \omega M}\right) G_{2}\left(e^{j \omega M}\right)\right|\right\} \leq 0.25 d B
$$

where:

$$
\begin{gathered}
0 \leq \omega \leq \pi /(2 M), \\
\left|G_{1}\left(e^{j \omega M}\right)\right|=1+2^{-N_{1}} \sin ^{4}(\omega M / 2), \\
\left|G_{2}\left(e^{j \omega M}\right)\right|=1+2^{-N_{2}} \sin ^{2}(\omega M / 2) .
\end{gathered}
$$

Considering $M>10$ the compensator parameters do not depend on $M$, [13]. Using the MATLAB simulation, and taking the condition (10), we can easily find the parameters $B_{1}$ and $B_{2}$, for $K=1, \cdots, 5$, shown in Table 1 . The number of required adders is equal to 9 , for $K=2, \cdots, 5$, and 6 for $K=1$. The maximum passband deviation is obtained for $K=4,(0.25 \mathrm{~dB})$, while the smallest one is for $K=1$, $(0.14 \mathrm{~dB})$.

Figure 1 illustrates the passband zooms for different values of $K$ and $M=15$.

The method is illustrated in the following example.

Example 1: We consider the value of $M=18$ and $K=5$. According to Table 1, the values of $B_{1}$ and $B_{2}$ are equal to $1 / 2$ and 1 , respectively. Figure 2 compares the magnitude responses of the compensated CIC and the corresponding CIC filter. The passband zooms show that the absolute value of the maximum passband

Table 1. Parameters $B_{1}$ and $B_{2}$ for wideband compensation.

\begin{tabular}{ccc}
\hline$K$ & $B_{1}$ & $B_{2}$ \\
\hline 5 & $1 / 2$ & 1 \\
4 & 1 & $1 / 2$ \\
3 & 1 & $1 / 4$ \\
2 & $1 / 2$ & $1 / 4$ \\
1 & $1 / 2$ & 0 \\
\hline
\end{tabular}




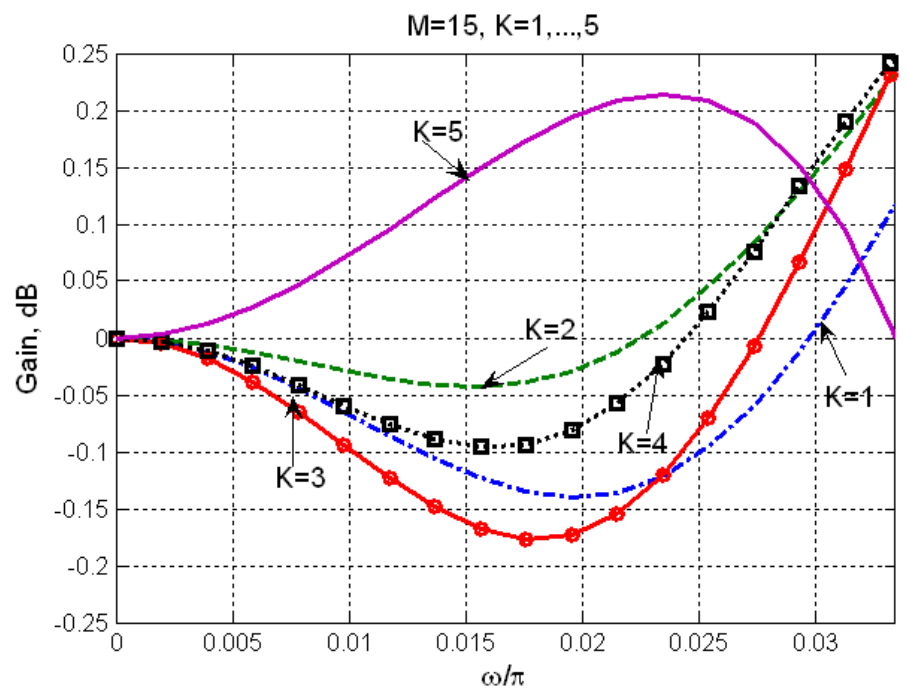

Figure 1. Passband zooms for compensated comb for $M=15$ and $K=$ $1, \cdots, 5$.

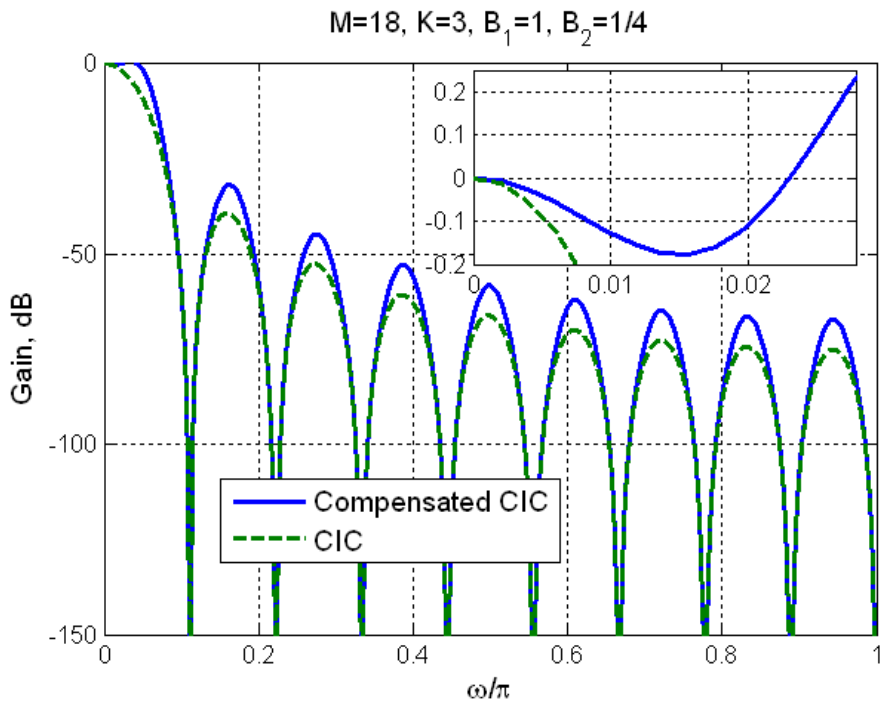

Figure 2. Magnitude responses of CIC and compensated CIC filters.

deviation is lesser than $0.23 \mathrm{~dB}$. The proposed compensator requires 9 adders.

The overall magnitude responses in Figure 2 confirm that the proposed compensator, despite the slight increase in the magnitudes of side lobes, does not deteriorate the attenuations in the folding bands.

\subsection{Narrowband Compensation}

We consider the passband edge $\omega_{p}=\pi /(8 M)$ for narrowband compensation and the following condition:

$$
\max _{N_{1}, N_{2}}\left\{20 \log 10\left|H\left(e^{j \omega}\right) G_{1}\left(e^{j \omega M}\right) G_{2}\left(e^{j \omega M}\right)\right|\right\} \leq 0.1 d B,
$$

where:

$$
0 \leq \omega \leq \pi /(8 M)
$$


and $G_{1}\left(e^{j w M}\right)$ and $G_{2}\left(e^{j w M}\right)$ are given in (11b) and (11c), respectively.

Applying the MATLAB simulation we got the values of parameters $B_{1}$ and $B_{2}$, shown in Table 2. The method is illustrated in Example 2.

Example 2: We consider values of $M=21$ and $K=5$ and the passband edge of $\omega_{p}=\pi /(8 M)$. Figure 3 shows the overall magnitude responses and the passband zooms for the compensated CIC and CIC filters. The absolute value of the passband deviation of the compensated CIC is lesser than $0.05 \mathrm{~dB}$. The compensator requires only three adders.

In next section are given some comparisons with the recently proposed compensators.

\section{Some Comparisons}

\subsection{Comparison with Method in [13]}

Consider $M=20$ and $K=5$. Figure 4 compares the passband zooms of the proposed compensator and compensator in [13]. The parameters in the proposed method are: $B_{1}=1 / 2, B_{2}=1$. The parameters of the compensator in [13] are: $B_{1}=1$ and, $B_{2}=2^{0}-2^{-2}-2^{-5}$, thus requiring total of 11 adders.

\subsection{Comparison with Method in [12]}

The compensator in [12] has two second order sections both with sine-squared

Table 2. Parameters $B_{1}$ and $B_{2}$ for narrowband compensation.

\begin{tabular}{ccc}
\hline$K$ & $B_{1}$ & $B_{2}$ \\
\hline 5 & 0 & 1 \\
4 & 0 & $1 / 2$ \\
3 & 1 & $1 / 2$ \\
2 & 1 & $1 / 4$ \\
1 & 1 & $1 / 8$ \\
\hline
\end{tabular}

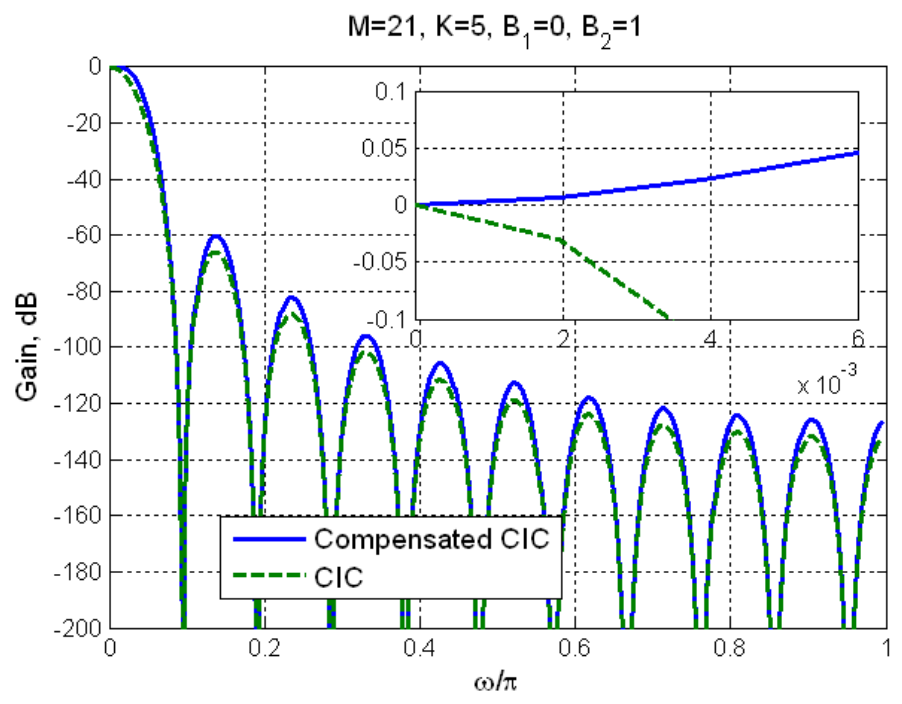

Figure 3. Magnitude responses of CIC and compensated CIC filters. 
magnitude responses with amplitudes of sine squared functions $B_{1}$ and $B_{2}$. The value of $B_{1}$ is equal to $2^{-3}$ for all values of $K$, while $B_{2}=(1+4(K-1)) / 16$. For the sake of comparison we consider $M=16$ and $K=5$. The compensator in [12] requires 7 adders. The passband zoom is shown in Figure 5.

\subsection{Comparison with Method in [9]}

The proposed compensator is compared with that in [9], taking $M=32$ and $K=$ 5. The result is shown in Figure 6. The compensator in [9] has 5 coefficients and requires 14 adders.

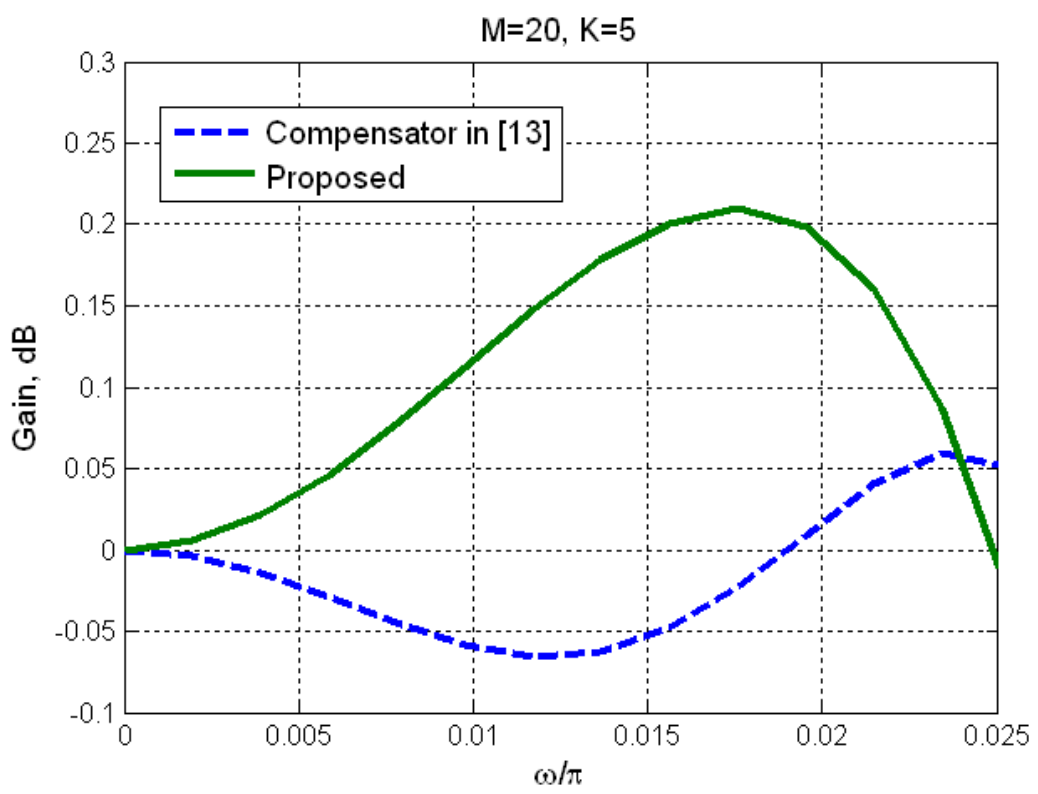

Figure 4. Comparison with method in [13].

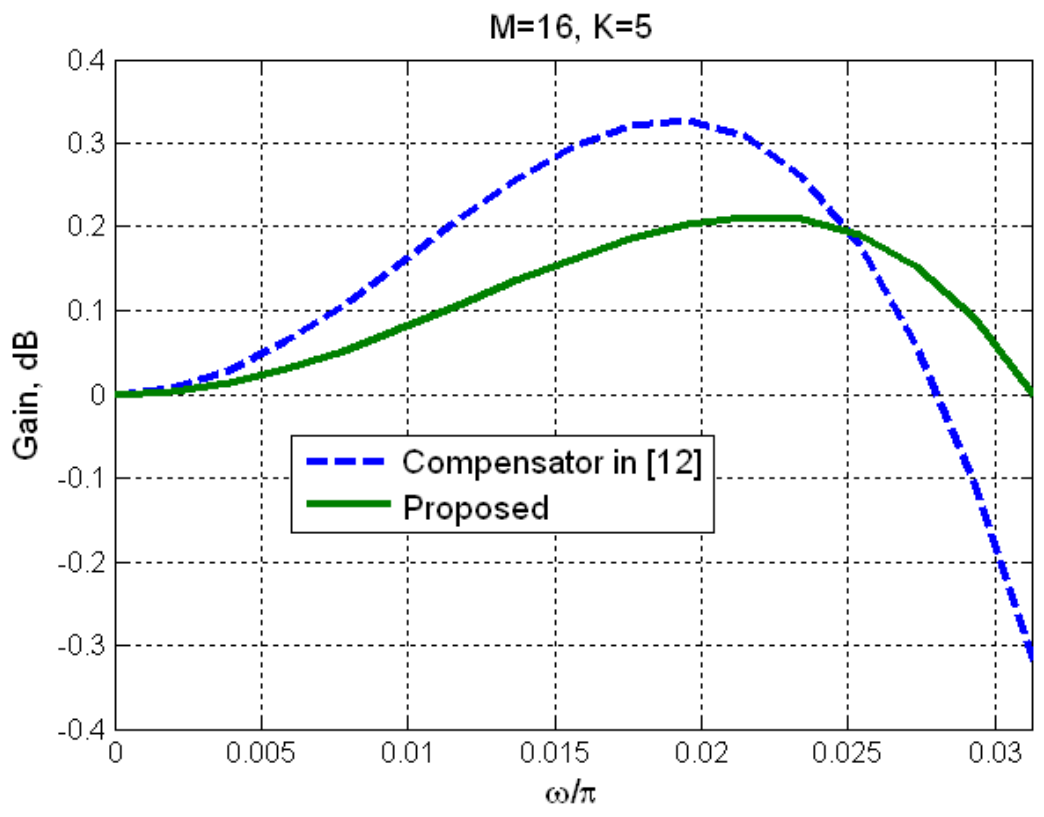

Figure 5. Comparison with method in [12]. 


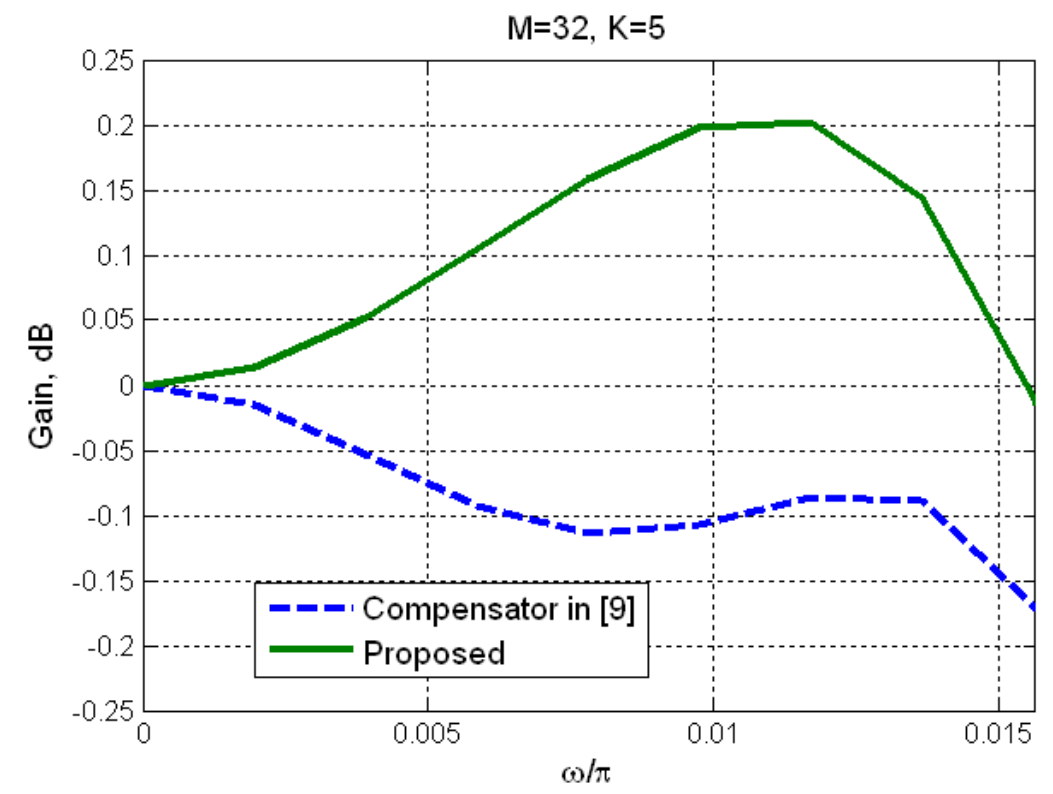

Figure 6. Comparison with method in [9].

\section{References}

[1] Mitola, J. (1995) The Software Radio Architecture. IEEE Communication Magazine, 33, 26-38. https://doi.org/10.1109/35.393001

[2] Hentschel, T. and Fettweis, G. (2000) Sample Rate Conversion for Software Radio. IEEE Communication Magazine, 38, 142-150. https://doi.org/10.1109/35.860866

[3] Jovanovic Dolecek, G. (Editor) (2001) Multirate Systems: Design and Application, IGP Hershey.

[4] Hogenauer, E.B. (1981) An Economical Class of Digital Filters for Decimation and Interpolation. IEEE Transactions on Acoustics, Speech and Signal Processing, 29, 155-162. https://doi.org/10.1109/TASSP.1981.1163535

[5] Fernandez-Vazquez, A. and Jovanovic Dolecek, G. (2012) Maximally Flat CIC Compensation Filter: Design and Multiplierless Implementation. IEEE Transactions on Circuits and Systems II: Express Brief, 59, 113-117. https://doi.org/10.1109/TCSII.2011.2180093

[6] Molnar, G. and Vucic, M. (2011) Closed-Form Design of CIC Compensators Based on Maximally Flat Error Criterion. IEEE Transactions on Circuits and Systems II: Express Brief, 58, 926-930. https://doi.org/10.1109/TCSII.2011.2172522

[7] Kim, S., Lee, W.C., Alm, S. and Choi, S. (2006) Design of CIC Roll-Off Compensation Filter in a W-CDMA Digital Receiver. Digital Signal Processing, 16, 846-854. https://doi.org/10.1016/j.dsp.2006.06.003

[8] Jovanovic Dolecek, G. (2009) Simple Wideband CIC Compensator. IET Electronics Letters, 45, 1270-1272. https://doi.org/10.1049/el.2009.1860

[9] Pecotic, M., Molnar, G. and Vucic, M. (2012) Design of CIC Compensators with SPT Coefficients Based on Interval Analysis. Proceedings of 35 th IEEE Int. Convention MIPRO 2012, IEEExplore, Opatija, Croatia, 123-128.

[10] Jovanovic Dolecek, G. and Fernandez-Vazquez, A. (2014) Trigonometrical Approach to Design a Simple Wideband Comb Compensator. International Journal for Electronics and Communications (AEUE), 68, 437-441.

https://doi.org/10.1016/j.aeue.2013.11.007 
[11] Romero, E.T. and Jovanovic Dolecek, G. (2013) Application of Amplitude Transformation for Compensation of Comb Decimation Filters. Electronics Letters, 49, 985987. https://doi.org/10.1049/el.2013.1492

[12] Jovanovic Dolecek, G. (2016) A Novel Comb Compensator with a Good Passband Deviation-Complexity Tradeoff. Proceedings of 2016 IEEE 59th International Midwest Symposium on Circuits and Systems (MWSCAS), IEEEXplore, Abu Dhabi, United Arab Emirates (UAE), 137-140.

[13] Jovanovic Dolecek, G., Garcia Baez, R., Molina Salgado, G. and de la Rosa, J. (2017) Novel Multiplierless Wideband Compensator with High Compensation Capability. Circuits, Systems and Signal Processing, 36, 2031-2049.

https://doi.org/10.1007/s00034-016-0398-0

Submit or recommend next manuscript to SCIRP and we will provide best service for you:

Accepting pre-submission inquiries through Email, Facebook, LinkedIn, Twitter, etc. A wide selection of journals (inclusive of 9 subjects, more than 200 journals)

Providing 24-hour high-quality service

User-friendly online submission system

Fair and swift peer-review system

Efficient typesetting and proofreading procedure

Display of the result of downloads and visits, as well as the number of cited articles

Maximum dissemination of your research work

Submit your manuscript at: http://papersubmission.scirp.org/

Or contactijcns@scirp.org 